best of the world's work has been done by subjects of status lymphaticus, but in the military field that work must have been fortunately adapted to the physical defects of the worker. Therefore, it seems not necessary for the clinician to condemn every subject showing status lymphaticus, but rather to find the subject's proper place in the military organization.

Speculation which seems legitimate suggests several topics of research that may be profitable from the clinical side.

No surgeon can afford to ignore the importance of status lymphaticus in the choice and administration of anesthetics.

The employment of injections of alien proteins and of arsphenamin should be undertaken with caution in subjects of status lymphaticus.

Further observations on the course of infectious diseases in subjects of status lymphaticus are called for. The idea that a fulminant course of meningitis or pneumonia is largely determined by status lymphaticus challenges the present conceptions of most clinicians and bacteriologists, but cannot be contested except by carefully accumulated clinical data.

The resistance to tuberculosis claimed by Bartels for subjects of status lymphaticus may be determined by the clinician and the pathologist. Not many clinicians will be prepared to accept the statement that Addison's disease occurs almost exclusively in subjects of status lymphaticus, but this question can be determined by the material collected in the Army.

Those who are interested in the doctrines of internal secretions may find profit in pursuing the relations of status lymphaticus to the ductless glands.

The neurologist meets phases of status lymphaticus notably in suicide, epilepsy and possibly in certain forms of mental disturbance. It would be interesting to know if any considerable proportion of patients

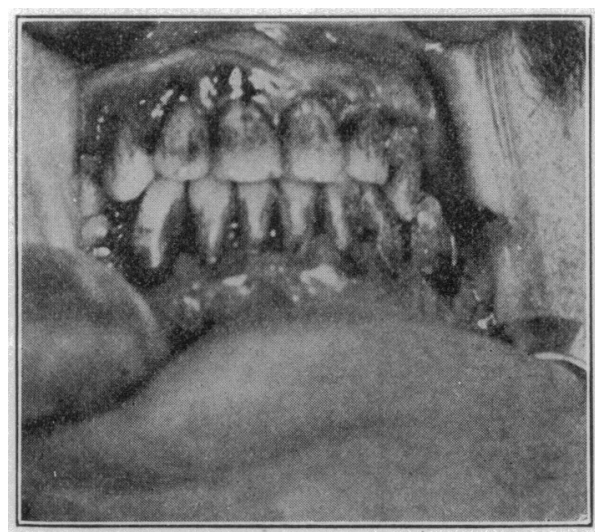

Fig. 1.-Appearance before operation.

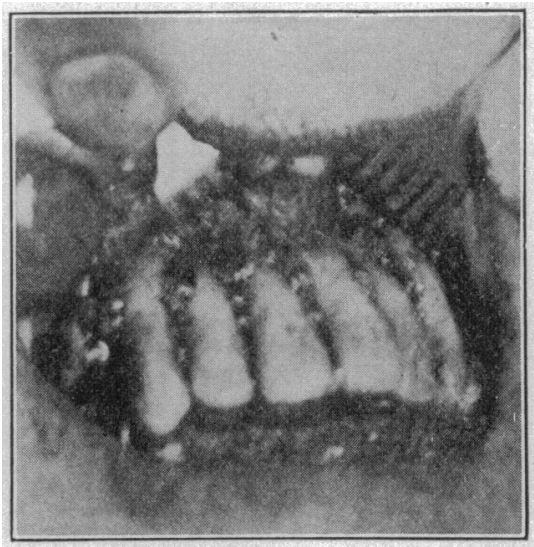

Fig. 2.-Flap lifted and retracted. with shell shock or other forms of nervous breakdown, with or without anatomic lesions, belong in the category of status lymphaticus. Given an overdelicate cerebral vascular system, one has an obvious predisposing condition to miliary or microscopic hemorrhages, such as occur in concussion and carbon monoxid poisoning. Almost the entire anatomic picture of status lymphaticus lends itself readily to the explanation of physical and mental breakdown on the battle field.

The irritable heart presents itself as a possible phase of the defective circulatory apparatus of status lymphaticus and one in which clinical observations on a large scale could be carried on.

All of these suggestions, however, must be offered with the greatest caution. Otherwise status lymphaticus may be lost in a maze of minor and ill defined clinical disorders of very doubtful relation to the very substantial anatomic alterations that appear in the bodies of pronounced cases. Such a fate seems to have overtaken neurofibromatosis at the hands of several continental writers, who have attempted to attach to this extremely specific anatomic condition all manner of disorders of the skin and nervous system.
The present conditions in the military service in America, when numerous races and classes are brought under the draft, seem to offer quite unique opportunity of determining the economic and military importance of definite status lymphaticus, and it is to this aspect of the subject, rather than to its possible ramifications, that the present note is directed.

Army Medical Museum.

\section{SUPPURATIVE GINGIVITIS WITH ALVEOLAR INVOLVEMENT}

\section{A NEW SURGICAL PROCEDURE *}

\section{ARTHUR ZENTLER, D.D.S.}

Associate in Oral Surgery, New York Post-Graduate Medical School and Hospital; Surgeon. Department of Oral Surgery, New York Throat, Nose and Lung Hospital (Columbia University,

Advanced Courses for Practitioners)

NEW YORK

It is my intention to deal with that phase of the disease commonly known under the confusing name of pyorrhea alveolaris, which as indicated by its histopathology is a purely surgical disease and must, therefore, be treated surgically, this alone promising an expedient and a permanent cure.

For the purpose of clear presentation, I shall briefly state that I prefer to call the diseases involving the gingivae and their soft and hard underlying and adjacent tissues by the generic name given them by Talbot, of gingivitis. Further, that for purposes of practical study, I classify gingivitis into superficial, hemorrhagic and suppurative.

The first class of the disease and the less advanced forms of the second and third classes are ordinarily amenable to proper dental treatment (scaling, polishing, etc.) in conjunction with correction of such local and constitutional disturbances as may be present.

The more advanced hemorrhagic cases, and especially the advanced suppurative cases, accompanied by degenerative processes of the alveolar bone and the soft tissues immediately covering it, are usually either traumatic, being due to long neglected accumulations of salivary calculi or other irritants (ill fitting fillings, inlays, crowns, bridges, etc.), or they are a manifestation of a disturbed metabolism due to such diseases as diabetes, syphilis, tuberculosis or chronic or acute

* Read before the Section on Stomatology at the Sixty-Ninth Annual Session of the American Medical Association, Chicago, June, 1918. 
metallic toxemias, when serumal calculi may or may not be found on the roots of the teeth, and, if present, will act as an additional irritant. Whether of traumatic origin or of metabolic or of both origins, these forms of gingivitis are oral foci of infection, to which

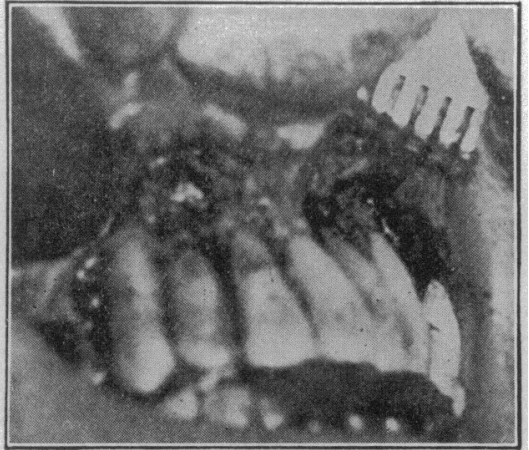

Fig. 3.-Appearance after removing all soft and hard diseased tissue. other systemic disturbances are attributed; but this phase of the sub-

by "cutting away as far, or a little beyond, the point to which the disease has reached, which generally means some cutting away of the margin of the alveolar process as well as the soft tissue." He speaks of there being "considerable variation in results following this treatment. The rule is, however, that something will be gained in reducing the depth of the pocket each time the overlying tissue is cut away."

From this it is seen that while the need of radically dealing by surgical methods with

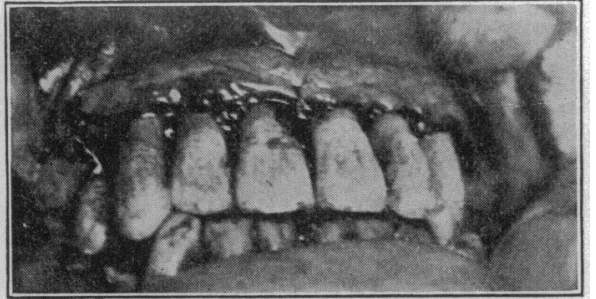

Fig. 4.-Flap replaced and sutured. these cases was recognized by Black, the result was not always a success and recurrence took place; hence the need of repeated ("each time") cutting away of the pocket.

Riggs, and others since his time, operated somewhat in the way described by Black. Robitzeck of Vienna, as long ago as 1862 , devised an operation for cases involving an area over several teeth, sometimes all the anterior region or

ject is not to be considered here, except for the purpose of stating that it is a sine qua non that the treatment of the oral condition must go hand in hand with the treatment of the general physical condition; neither can be brought to a successful issue without a rational treatment of both.

A typical report ${ }^{1}$ of microscopic examination of specimens of tissues obtained from areas operated on reads as follows:

The exposed connective tissue is of sluggish granulation type, heavily infiltrated by some polynuclear and many round and plasma cells. These last cells are so dense that the stroma is obscured throughout its length inward from the epithelium. The epithelium is not remarkable except at points where it is necrotic and desquamating. There are many congested capillaries and considerable extravasation by red blood cells throughout, and several spicules of alveolar bone are seen

This shows that the condition present is of such character as clearly to indicate that nothing short of radical removal of all the involved soft and hard structures, going well into healthy surroundings, will render the part receptive of regenerative processes and insure an ultimate permanent cure. It is clear that an essential factor in operating on any part is that its approach be unobstructed, so that before terminating the operation, the surgeon may be certain of having actually removed all that is diseased; it is seldom, if ever, when this is not accomplished, that recurrence does not take place. This probably explains the reasons why, in advanced cases of socalled pyorrhea, very few permanent cures are effected with the treatments as generally practiced at present.

G. V. Black ${ }^{2}$ speaks of the surgical treatment of pockets, describing a method of eradicating the pocket

1. Meeker, L. H.: From the Pathological Laboratory of the New York Post-Graduate Medical School and Hospital.

2. Black, G. V.: A Work on Special Dental Pathology, Chicago, Medico-Dental Publishing Company, 1915, chapter on Treatment of Chronic Suppurative Pericementitis. the posterior region or even both regions, consisting in excising with a straight incision through the soft tissues down to the alveolar process, all the abnormally appearing gum tissue. A scraping of the exposed alveolar bone followed the excision of the soft tissue, and the patient was dismissed. In cases in which cures were effected with this treatment, the result of the straight incision was such as to leave a very unsightly aspect of the parts.

After practicing Robitzeck's operation for a number of years, I have found that it was very useful in cases of advanced hemorrhagic gingivitis and often in cases of not very advanced suppurative gingivitis. I found, however, that in somewhat modifying his technic I could obtain a more pleasing ultimate appearance and a local condition more easily kept clean.

My modification consisted in excising the diseased gum tissue, instead of by a straight incision, by following the original festoons of the gums, going well into the healthy tissue, thus leaving behind a scalloped edge, which I packed tight with a strip of folded iodoform gauze one-eighth inch wide. The ulterior healing would result in an appearance of the gum very much as if it had never been diseased, and with no signs of an operation noticeable; the gingival papillae would be practically restored.

This operation, however, was purposeless in advanced suppurative cases in which the involvement 
of the soft and alveolar tissue was extensive, and recognizing the cause of the failure to be the lack of proper approach to all the involved area, I devised the following operation:

\section{TECHNIC OF OPERATION}

The patient is prepared as for all oral surgical operations, performed under procain-epinephrin conductive anesthesia.

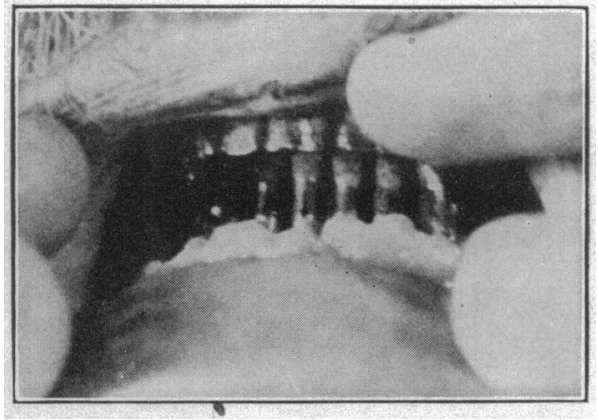

Fig. 7.-Typical appearance of lower anterior region before operation in an extensive case of suppurative gingivitis in a patient aged 55 .

The part to be operated on (Fig. 1) is swabbed with tincture of iodin, and a flap is lifted by making two parallel incisions, starting at the cervical free border of the gum, and carrying them apically to the apical region each side of the area over the tooth or teeth involved. The incisions are made so as to penetrate the mucosa and submucosa, and to include the periosteum. This, with the gum tissue overlying it, is lifted with the raspatory, retracted, and held in position by the assistant (Fig. 2). Thus the diseased part is well exposed, and with suitable curets and knives, all the inflamed, infected, granulomatous tissue found, between and surrounding the roots of the teeth, is removed.

Next, with delicate chisels and mallet, the alveolar plate is chiseled away from around the denuded portions of the roots, so as to insure the removal of any and all infected bone covering the roots, which are then well curetted. The rough edges of the remaining alveolar bone covering the roots are well smoothed so as to form an even surface with them (Fig. 3).

The flap is then released, and its inner aspect is inspected for possible adhering inflammatory and granulomatous

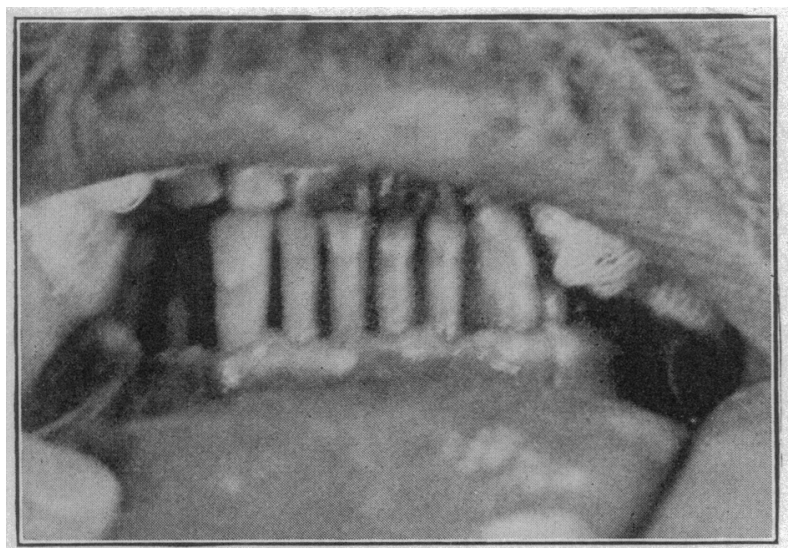

Fig. 8.-Same patient as is shown in Figure 7, four months after operation.

tissue, which is thoroughly curetted away, disturbance of the remaining healthy periosteum being carefully avoided. The parts are washed with physiologic sodium chlorid solution, swabbed with a 50 per cent. solution of the official tincture of iodin, the wound surfaces are freshened up, and the flap is replaced in position and sutured (Fig. 4).

The sutures are taken at each end of the incision, one near the cervical end and the other near the apical end. When

the operation is performed over an area covering more than four teeth, it is well to take one or two additional sutures between the teeth, securing the approximation, for instance, of a labial flap to the palatal gingiva. The parts of the free edge of the sutured flap that appear to be necrotic from lack of nutrition, because of the long existing interposed infection, are cut away with sharp scissors, leaving a clean cut which, when healed, presents a well adhering surface, surrounding the root of the tooth. Before the patient is dismissed, the parts are again swabbed with tincture of iodin and covered with a strip of iodoform gauze, which is changed once or twice at intervals of twenty-four hours. The sutures are removed on the fourth or fifth day, and unless the operation was attempted in an extensively necrotic case, in which removal of the tooth or teeth should have been practiced, there will in time be new bone formation around the roots, complete reattachment of the soft tissues, and quite loose teeth will become firm and stay firm (Figs. 5, 6, 7 and 8 ).

\section{COMMENT}

It is needless for me to say that it is advisable that the mouth be placed in as hygienic a condition as possible before the operation, all exgingival irritants, such as salivary calculi or ill fitting fillings, inlays, crowns, etc., being removed from the area to be operated on, and preferably from the entire mouth. In cases in which, for some reason or other, this was not done prior to operation, the patient must be warned

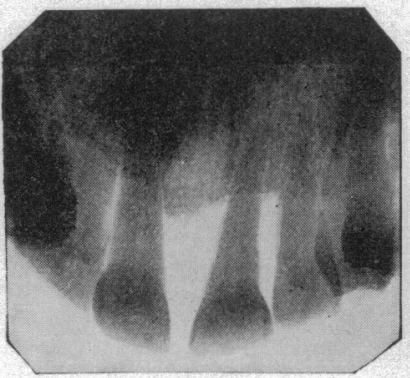

Fig. 9.-Amount of alveolar re. sorption over upper anterior region in patient on whom opera. tion was performed, shown in Figure 5 .

that it ought to be done as soon as the parts have sufficiently recuperated to permit handling them, and the rest of the mouth, for such purposes. This is usually from ten to twelve days after the operation. The patient may then resume the rational care of teeth and gums with tooth-brush and tooth-powder, etc., on the operated area. In the rest of the mouth this need not be interrupted at all, except in rare cases, for one day after operation.

There is usually no discomfort following the operation. At times there is some swelling and pain, after the effect of the procain wears off. The application of an icebag fifteen minutes to the hour, for two or three hours, usually brings prompt relief from pain and reduces the swelling.

All areas of the mouth, without exception, can be operated on as described above. I have extended the operation over as many as six adjoining teeth; but it is advisable when there is a great deal of suppuration in the entire region not to operate' at one time over more than three or four teeth, in order to avoid opening up too large an area for septic absorption.

Whenever there are devitalized teeth in the area to be operated on, these must first be thoroughly treated; root canals must be thoroughly, aseptically filled. Apicoectomies may be performed at the same time as the operation for the suppurative peridental 
condition. In multirooted teeth this is not always practicable and, therefore, such nonvital teeth, with periapical and peridental pathologic conditions, would better be removed.

As in all other oral surgical operations, the roentgenogram (Figs. 9 and 10) is an indispensable adjunct in diagnosis. It is my experience that when the roentgenogram shows that one side of the root is still supported by investment tissue, the case is a good operative risk. Even in cases in which apparently, from the rocntgenogram, the investment tissue is lost almost to around the apical region, the tooth, however, responding to the ethyl chlorid thermal test, the operation was sticcessfully performed in a small number of cases attempted for purposes of experimentation.

\section{ADVANTAGES OF THE METHOD}

Full exposure of the entire affected area is obtained, and hence facility for a thorough operation. Once the operation has been correctly performed and the patient dismissed after a few days of observation, the parts formerly harboring infection are totally obliterated, and the patient is able with only reasonable care for clcanliness of the mouth to enjoy health in the parts formerly diseased. Some cases that would. be pronounced incurable by the ordinary methods now in vogue for treating so-called pyorrhea alveolaris might be successfully treated by this surgical method.

S. West Forticth Strect.

\section{ABSTRACT OF DISCUSSION}

DR. Arthur 1). Bi.Ack, Chicago: I approve of almost all Dr. Zentler prescintcd, yet I cannot see the advantage of hringing back a flap and making a complicated operation instead of simply cutting away the overlying tissue. Scraping the surface of the root and bringing the soft tissue over it is just what we should not do. If the cementum is dead no soft tissue should be left overlying it. It is very easy to remove all of the detached tissue if the pockets are on the labial, buccal or lingual surfaces of roots, but when the pockets are between the teeth, it is necessary to cut away not only the tissue which is detached from the root, but also some of the soft tissue and the bone on the buccal and lingual sides of the interproximal space, in order to promote cleanliness. In most cases it is necessary to cut away a little of the edge of the bone, because the detachment from the root surface is usually deeper than the crest of the bone over the particular area. No matter what form of treatment has been employed, many operators have been deceived by the fact that the tooth, which may have hecn quite loose, has become much less so, and they have thought there has been a reattachment of the peridental memhrane. The amount of destruction of the peridental membrane and the looseness of the tooth are not necessarily definitely related one to the other. Inflammations involving the peridental tissues cause the fibers to lose tone so they do not hold the tooth tightly in its socket. Following almost any treatment by which the inflammation is reduced, the teeth have less motion within a kew days. This does not mean regeneration of tissue, but only that the fibers have regained their tone.

Dr. Moorehcad might tell you of the experiments on dogs in which he and Dr. Noyes attcmpted to cut the peridental mcmbrane away from the cementum. In each case they found, on microscopic examination, that the cementum had not been stripped; that a considerable amount of the soft tissue remained attached to the cementum. When cut in this way the peridental membrane will heal quite as readily as any other soft tissue.

Dr. George Edward Fell, Chicago: I have looked on this as a case of infection which must be overcome and in which the mouth has to be placed in such condition that reinfection callnot take place. It is evident that most of our cases of gingivitis can be cured if proper methods are employed and carried out radically.

Dr. Thomas L. GrLmer, Chicago: Dr. Zentler has indicated that bone is built up on the sides of the roots of these teeth. I would like to have him state rather definitely, if he will, what is the nature of the union between root and tooth. Is it vital, or if nonvital, is it a union similar to that found in implanted teeth; that is, an absorption of the cementum and a building in of the alveolar process?

Dr. Truman W. Brophy, Chicago: When a part is in an abnormal state, put it to rest. So that the very best thing to do in the treatment of a tooth that is inflamed, as described in this case, would be to put the parts to rest. Band the teeth and fix them so they will not be moving about after the operation is done. In the second place, the tooth root that has been deprived of its pericementum by this suppurative process should be opened and the diseased tissue, together with the end of the root, removed. A few days ago a dentist came with his patient, for whom he removed a first lower molar some weeks before. The patient was still in a great deal of pain and distress. He had remover the tooth, the roots of which had two great abscesses. The tooth came away, but the abscesses remained. After curetting the alveoli and removing the diseased tissue the man was very comfortable in a few days.

Precisely the same condition is present in these cases where it becomes necessary to penetrate the alveolar process and remove diseased tissue together with the end of the root. The surgeon would not be regarded as a very wise man or a very conservative practitioner if he would amputate a leg because his patient had an osteomyelitis. The position is exactly the same regarding the teeth. What the doctor showed us here in the excision of diseased teeth and removal of diseased teeth is correct, and by and by there will be sufficient enlightenment on this question so that so many teeth will not be extracted unwisely.

In regard to the entrance of germs after cutting off the root of the tooth and subsequent treatment, you do not try to sew up the socket or gum tissue after you extract teeth. You allow granulation tissue to fill the alveoli, and after a while you have a well-developed alveolar process. The same is true after excising diseased tissue and the end of the root. The cavity should be left precisely as a tooth socket is left following extraction of a tooth. We all know how these sockets have filled in, leaving a good, round, smooth surface. To amputate the apex of a tooth root is right, but to make a flap and close the cavity by stitches, leaving a place where another abscess may form, should not be done. I would make a funnel-shaped opening, remove the diseased root and diseascd tissue, getting it free and clean, and then keep it open until granulations fill the cavity and until the healing is complete. In that way you will not have recurrence of the disease.

Dr. Frederick B. Moorehead, Chicago: The fundamental underlying question in the type of infection suggested by Dr. Zentler is with the hard tissue and not with the soft tissue. The seat of pathology in these cases is in the hard tissue. The most important question in this whole matter is that of a normal circulation. Where you find a proliferating endarteritis associated with a chronic heart or kidney no amount of local treatment will avail. These infections will not clean up in the absence of a healthy circulation, which, of course, cannot be had unless the blood vessels are normal. Any method, therefore, that has for its object the saving of teeth under these special conditions must be contingent on the general condition of the patient. One may undertake to save teeth when the general conditions are good, while he would remove similar teeth where general conditions were ? vor. The condition of the circulation and the general repatutive power of the patient are paramount factors in guiding is between our efforts to save teeth and their immediate re::ivval. The tooth has no analogue and one cannot, therefore, make a comparison between a tooth and other bony structures of the body. One half of a tooth root may be vital because of its investment in peridental membrane, while the other half may be a foreign body. The portion of the tooth root which has been deprived of its peridental membrane through infection 
will act as a foreign body and not be sequestrated. This is not true of any other bone infection in the body. For example, one half of a long bone may be devitalized through infection and be thrown off as a sequestrum. There can be no organic union between a tooth root which has lost its peridental membrane and the surrounding bone or soft tissue. It would seem, therefore, that these elemental fundamental facts must be our guide in undertaking the management of infected teeth.

Dr. E. S. Fuller, Dayton, Ohio: Some years ago, in order to obtain better access for cleaning the root surface of a lower incisor, I made an incision along the axial line of the tooth, cleaned the surface and sutured the gum flaps together. The stutures pulled out and the root surface became exposed. This I thought unfortunate at the time, but now I think it was the best thing which could have happened. During the past three years I have been treating these "pyorrhea pockets" by removing the overlying gum tissue in all cases, excepting those where the pockets were shallow enough to become obliterated by the reduction of the gingival inflammation following the removal of deposits and the cleaning of the root surface. Many cases are seen showing a deep "pyorrhea pocket" on both the mesial and distal proximal surfaces of a tooth-say a bicuspid, for example-the adjoining teeth not being affected. In these cases I frequently extract the diseased tooth rather than resect away the gum tissue from the interproximal space, as a horizontal resection would destroy the attachment of the tooth not involved.

Dr. Arthl一 Zextler, New York: To understand the character of the union obtained we would have to secure a section embracing all the tissues concerned and submit the whole for histologic examination. It is not easy to obtain human subjects for this. Animal experimentation may be used for the purpose. I believe, however, that we do not have the same conditions in animal as in human mouths. Practical examination shows a union, in operated cases, difficult to penetrate with fine probes. Only by exposing the entire involved area can one eradicate the disease. Simply cutting away the pocket has not proved permanent.

Where I operate at the same time for peridental and periapical infection, I make the incisions as described in my operation. A month after removing the sutures there is no sign left of these incisions, while with the semicircular incisions for apicoectomies cicatrices are present for a long time. One need not fear to suture the flap in either of these operations, provided the operation is done as aseptically as it should be done. I never had an ulterior infection due to suturing. I thought it understood that only healthy tissue is allowed to remain, and in speaking of reattachment $\mathbf{I}$ did not mean a reattachment of pericementum, but a reattachment of soft tissues that had been lifted and then replaced carefully, saving the healthy periosteum. Twice I operated in cases previously splinted. The operations were failures. It was due, I believe, to the lack of normal motion. It interferes with circulation and deprives the part of proper nutrition.

It is true that the fundamental pathology is with the hard tissues and not with the soft, and that is precisely why I felt the necessity of lifting a flap and of getting to the hard tissues underneath. That is why I chisel away a strip of the involved alveolar plate as well as curetting the soft involved tissue which lies over it and surrounds the roots. That is why I pronounced it a poor operative risk to operate when all investing tissue is gone, and advise in such cases removal of teeth.

As to the general condition of the patient, I think it is understood that if it cannot be controlled one need not attempt local treatment expecting success. Both must go hand in hand. The local circulation will be improved by the operation if we remove the infected parts. The general circulation must be taken care of by the patient's physician.

Dr. Brophy: Is there not some vitality left in the cementum?

Dr. ZentLeR: Wherever there is cementum left on a portion of the root that had prior to operating a detachment of the investing tissue, it is an open question in my mind whether this cementum is not able to throw off further cells into newly created healthy surroundings to form some sort of attachment. Where suppuration has intervened I do not believe there will be any live cementum. We get a reattachment of healthy tissue to healthy tissue in approximating them. You need not fear at all to remove some of the healthy tissue if you must do it in order to eradicate all the diseased tissue. The lost healthy tissue will be replaced the same as in any other surgical procedure.

\section{POSSIBILITIES OF FRACTIONAL GASTRIC ANALYSIS *}

\section{MARTIN E. REHFUSS, M.D. PHILADELPHIA}

Fractional gastric analysis has for its purpose the determination of the evolution of gastric digestion. The necessity for such extended technic is justified by the fact that gastric digestion is marked by a series of constantly changing phases, so that it may now be definitely stated that an examination of any single phase of the digestive curve gives no information as to what has preceded or what will follow that phase. The most marked changes may occur in comparatively short intervals, so that findings at one period may be doubled or tripled at another period. The main point, however, is the essential fact that gastric digestion is the response of the organ to a definite stimulus, which, in health, evolves in a perfectly methodical and coordinated manner, and in disease evolves with every possible variation. In order, therefore, to interpret gastric disease, we must study the elements governing the response of the organ in health.

\section{NORMAL DIGESTION}

From a study of normal digestion with every form of foodstuff, which the entire staff of the chemical department of the Jefferson Medical College was engaged, we arrived at the following conclusions:

Human gastric digestion is divided into a series of recurring cycles which, for the want of better names, we have called the digestive and interdigestive cycles. These succeed each other in health with regularity and represent most perfectly the normal gastric mechanism. The digestive cycle is that portion in response to the ingestion of food of any kind and evolves in a perfectly coordinated manner. There is practically no latent period and there are well marked psychic and chemical phases to secretion. The psychic secretion is very considerable, as we have been able to show, and in health it probably averages 250 c.c., lasting from sixty to eighty minutes and being marked, according to our recent studies, by changes in environment, fatigue, etc. The total acidity averages 97.2 in our series and it is markedly reduced, but does not disappear with atropin administration. The chemical secretion probably cómmences early but reaches its height later and completes digestion. During this active period, we notice (1) the relaxation of the perisystole and the inauguration of peristalsis, and (2) a change from the resting secretion to a pronounced secretion of much higher acidity. Furthermore, we can detect certain regulating mechanisms such as tryptic or duodenal regurgitation regulating acidity. This cycle is constantly changing as each factor comes into play. It is followed by the interdigestive phase characteristic of normal digestion.

* Read before the Section on Gastro.Enterology and Proctology at the Sixty-Ninth Annual Session of the American Medical Association, Chicago, June, 1918 